TRANSACTIONS OF THE

AMERICAN MATHEMATICAL SOCIETY

Volume 351, Number 1, January 1999, Pages 271-283

S 0002-9947(99)02169-8

\title{
DENSITY DOUBLING, DOUBLE-CIRCULANTS, AND NEW SPHERE PACKINGS
}

\author{
ALEXANDER VARDY
}

\begin{abstract}
New nonlattice sphere packings in dimensions 20, 22, and 44-47 that are denser than the best previously known sphere packings were recently discovered. We extend these results, showing that the density of many sphere packings in dimensions just below a power of 2 can be doubled using orthogonal binary codes. This produces new dense sphere packings in $\mathbb{R}^{n}$ for $n=25,26, \ldots, 31$ and $55,56, \ldots, 63$. For $n=27,28,29,30$ the resulting packings are denser than any packing previously known.
\end{abstract}

\section{INTRODUCTION}

The problem of constructing dense arrangements of nonoverlapping spheres in the $n$-dimensional Euclidean space $\mathbb{R}^{n}$ dates back to the work of Newton and Gauss [11], and was mentioned by Hilbert [12] on his epochal list of open problems in mathematics. In the study of sphere packings in $\mathbb{R}^{n}$, a particular effort has been devoted to low dimensions. The laminated lattices [5, pp. 157-180] were until recently the densest packings known in dimensions $n \leq 29$, except for $n=10,11,12,13$. For $n=12$ the Coxeter-Todd [7] lattice $K_{12}$ is the densest known packing. For $n=10,11,13$ nonlattice packings denser than the laminated sequence were found by Leech and Sloane $[17,18]$ in 1970, using constructions from nonlinear binary codes. Notwithstanding the vast body of research on dense sphere packings in recent years, see Conway and Sloane [5] and references therein, no new sphere packings in dimensions $n \leq 29$ that are denser than the already known ones, were reported in the following 25 years.

In a recent work [24], we have described a 20-dimensional nonlattice packing $\mathcal{J}_{20}$ which is denser than any packing in $\mathbb{R}^{20}$ previously known. This packing was originally obtained through a new density-doubling technique based on the use of two orthogonal binary codes. Subsequently, Conway and Sloane [6] have recast the construction of [24] as the first case of the antipode construction for sphere packings, similar to the anticode construction for error-correcting codes [20, p. 548]. This led them to the discovery [6] of new packings in dimensions 22 and 44-47 that are denser than previously known.

Since the preprints of $[6,24]$ became available about three years ago, a substantial effort has been devoted to the search for additional record-density antipode sphere packings. However, so far, all the attempts at extending the antipode construction

Received by the editors January 1, 1997.

1991 Mathematics Subject Classification. Primary 52C17, 11H31, 94B15.

This research was supported by the Packard Foundation Fellowship and by a grant from the National Science Foundation. 
beyond the results of $[6,24]$ have been unsuccessful. On the other hand, we show in this paper that the original density-doubling construction of [24], that was presented therein for sphere packings in dimensions $n \leq 24$, can be easily extended beyond 24 dimensions. As it turns out, the density-doubling construction is particularly successful when $n$ is just below a power of 2 . Using this construction, we produce herein new dense sphere packings in $\mathbb{R}^{n}$ for $n=25,26, \ldots, 31$ and $55,56, \ldots, 63$. In particular, for $n=27,28,29,30$, we obtain sphere packings that are denser than any previously known packing in the corresponding dimensions, as detailed in what follows.

For $n=27$, the densest known packing [5, p.157] is the laminated lattice $\Lambda_{27}$ with center density $1 / 2$, while the new packing $\mathcal{J}_{27}$ has center density $1 / \sqrt{2}$. Until recently, the laminated lattices $\Lambda_{28}, \Lambda_{29}$ with center density $1 / 2$ were the densest known packings in dimensions $n=28,29$. In a development contemporaneous with our work, Roland Bacher [1] has constructed new lattice packings in these dimensions with center density $1 / \sqrt{3}$. However, the nonlattice sphere packings $\mathcal{J}_{28}$ and $\mathcal{J}_{29}$ described in this paper are even denser: $\mathcal{J}_{28}$ has center density 1 and $\mathcal{J}_{29}$ has center density $1 / \sqrt{2}$. Finally, the densest known [5, p.220] packing in dimension $n=30$ is the cross-section $Q_{30}$ of the Quebbemann [21] lattice $Q_{32}$ with center density $3^{13} \sqrt{3} / 2^{22}=0.6584 .^{1}$, whereas the new packing $\mathcal{J}_{30}$ has center density 1.

We note that some of the other packings constructed herein are also quite dense, although they are slightly less dense than the existing records in the corresponding dimensions.

Finally, we point out that Bierbrauer and Edel [4] have very recently constructed a new sphere packing in dimension $n=18$ with center density $3^{9} / 2^{18}=0.07508$.., which is denser than the previously best known packing $\Lambda_{18}$, whose center density is $\sqrt{3} / 24=0.07217 \ldots$ The methods used in [4] are similar to those of [24] and the constructions described herein.

The rest of this paper is organized as follows. In the next section we review the density-doubling construction of [24] and show that it extends beyond 24 dimensions. In Section 3, we apply this construction to sphere packings in dimensions $n \leq 31$. The results rely on our analysis of the duals of certain double-circulant binary codes of Karlin [13]. In Section 4, we consider sphere packings in dimensions $n \leq 63$. We conclude in Section 5 with a new table of the densest known sphere packings in dimensions $n=1,2, \ldots, 48$.

\section{Density doubling And orthogonal Binary Codes}

We start with some notation, as in [24], included here for completeness. Let $S$ be an open $n$-dimensional sphere of radius $\rho$. An infinite set $\Lambda$ of vectors $x_{1}, x_{2}, \ldots$ in $\mathbb{R}^{n}$ is a sphere packing if the translates $x_{1}+S, x_{2}+S, \ldots$ are pairwise disjoint. It is a lattice packing if the vectors $x_{1}, x_{2}, \ldots$ form a group under addition in $\mathbb{R}^{n}$.

\footnotetext{
${ }^{1}$ Lattices with the same density can be obtained as cross-sections of the Elkies [8,9] lattices $E_{32}$ and $E_{32}^{*}$; although $Q_{32}, E_{32}$, and $E_{32}^{*}$ are not equivalent, they and their cross-sections have the same density [10]. Quebbemann has described in [22] another (inequivalent) lattice $Q_{32}^{\prime}$ with the same density. Finally, one more lattice $B_{32}$ with the same density as $Q_{32}, Q_{32}^{\prime}, E_{32}$, and $E_{32}^{*}$ was constructed by Bachoc [2]. We shall, however, refer to the (chronologically first) construction of Quebbemann [21] throughout this paper.
} 
W.l.o.g. it is assumed that $2 \rho$ is the minimum distance between two points of $\Lambda$. Then the density of $\Lambda$ is the fraction of the space covered by the spheres, and the center density $\delta(\Lambda)$ is the density divided by $V_{n}=\pi^{n / 2} / \Gamma(1 / 2 n+1)$, which is the volume of a unit sphere in $\mathbb{R}^{n}$. The integer lattice $\mathbb{Z}^{n}$ consists of all the vectors in $\mathbb{R}^{n}$ with integer coordinates. The volume of a packing $\Lambda$, denoted $V(\Lambda)$, is defined as the ratio of the number of points of $\mathbb{Z}^{n}$ to the number of points of $\Lambda$ contained in a sphere of radius $R$, in the limit as $R \rightarrow \infty$ (provided the limit exists). We let $\mu(\Lambda)=4 \rho^{2}$. The center density may then be found via $\delta(\Lambda)=\mu(\Lambda)^{n / 2} / 2^{n} V(\Lambda)$.

The set of binary $n$-tuples is denoted $\mathbb{F}_{2}^{n}$. For $x, y \in \mathbb{F}_{2}^{n}$, the Hamming distance $d(x, y)$ is the number of positions where $x$ and $y$ differ. The Hamming weight of $x \in \mathbb{F}_{2}^{n}$ is given by $w t(x)=d(x, \mathbf{0})$, where $\mathbf{0}$ denotes the all-zero $n$-tuple. The all-one $n$-tuple is denoted $\mathbf{1}$. An $[n, M, d]$ code $\mathbb{C}$ is a subset of $\mathbb{F}_{2}^{n}$ of cardinality $M$, such that $\min _{x, y \in \mathbb{C}} d(x, y)=d$. A linear $(n, k, d)$ code is a subspace of $\mathbb{F}_{2}^{n}$ of dimension $k$ and minimum distance $d$. Two codes $\mathbb{C}_{1}$ and $\mathbb{C}_{2}$ are said to be orthogonal if $(x, y)=0$ for all $x \in \mathbb{C}_{1}$ and $y \in \mathbb{C}_{2}$, where $(\cdot, \cdot)$ stands for the inner product in $\mathbb{F}_{2}^{n}$. The subspace of $\mathbb{F}_{2}^{n}$ consisting of all the vectors that are orthogonal to a code $\mathbb{C}$ is the dual code of $\mathbb{C}$, denoted $\mathbb{C}^{\perp}$.

We shall make use of the coordinate array of a point $x=\left(x_{1}, \ldots, x_{n}\right) \in \mathbb{Z}^{n}$, as defined by Leech and Sloane [18]. The coordinate array of $x$ is obtained by writing the binary expressions of the coordinates $x_{i}$ in columns, using complementary notation for the negative integers. The first, second, third, ... rows of the coordinate array are called the 1's, 2's, 4's, ... rows respectively. Given a finite sequence of binary codes $\mathbb{C}_{0}, \mathbb{C}_{1}, \ldots, \mathbb{C}_{m-1}$, consider a packing $\Lambda$ consisting of all the points $x \in \mathbb{Z}^{n}$ which satisfy the following property: the $2^{i}$-s row in the coordinate array of $x$ is a codeword of $\mathbb{C}_{i}$ for $i=0,1, \ldots, m-1$. We shall use the notation

$$
\Lambda=\mathbb{C}_{0}+2 \mathbb{C}_{1}+\cdots+2^{m-1} \mathbb{C}_{m-1}+2^{m} \mathbb{Z}^{n},
$$

to describe such a packing. The expression in (1) is similar to Construction $\mathrm{C}$ of Leech and Sloane [18]. We shall refer to $\Lambda$ as a Construction $\mathrm{C}$ coordinate-array packing, or simply a coordinate-array packing. It is not difficult to see that

$$
\begin{aligned}
V(\Lambda) & =\frac{2^{m n}}{\prod_{i=0}^{m-1}\left|\mathbb{C}_{i}\right|}, \\
\mu(\Lambda) & \geq \min \left\{d_{0}, 4 d_{1}, \ldots, 4^{m-1} d_{m-1}, 4^{m}\right\},
\end{aligned}
$$

where $d_{i}$ denotes the minimum distance of $\mathbb{C}_{i}$. The codes $\mathbb{C}_{0}, \mathbb{C}_{1}, \ldots, \mathbb{C}_{m-1}$ are often chosen such that $d_{i}=4^{m-i}$, in which case $\mu(\Lambda)=2^{2 m}$ and $\delta(\Lambda)=\prod_{i}\left|\mathbb{C}_{i}\right| / 2^{n}$.

It is well known (and trivial) that if $n \geq 4 \mu(\Lambda)$, then the density of $\Lambda$ can be doubled by adjoining to it the translate $\Lambda^{\prime}=(1 / 2, \cdots, 1 / 2)+\Lambda$. Indeed, for any $\alpha \in \Lambda$ and $\beta \in \Lambda^{\prime}$, we have $\|\alpha-\beta\|^{2} \geq n(1 / 2)^{2} \geq \mu(\Lambda)$, where $\|\cdot\|$ denotes the Euclidean norm. Thus the union $\Lambda \cup \Lambda^{\prime}$ has the same packing radius $\rho$ as $\Lambda$, and contains twice as many points. This simple density-doubling technique is used, for example (cf. [5, p.119]), in constructing the Gosset lattice $E_{8}$ from two copies of the checkerboard lattice $D_{8}$ with $\mu\left(D_{8}\right)=2$.

We now show that under certain conditions the density of a coordinate-array packing $\Lambda=\mathbb{C}_{0}+\cdots+2^{m-1} \mathbb{C}_{m-1}+2^{m} \mathbb{Z}^{n}$ can be doubled, or at least increased, even if $n<4 \mu(\Lambda)$. First, assume that all the codewords of the code $\mathbb{C}_{1}$ have even Hamming weight, and define $\mathbb{C}_{1}^{*}=(1,0, \ldots, 0)+\mathbb{C}_{1}$. Thus $\mathbb{C}_{1}^{*}$ has the same parameters $\left[n, M_{1}, d_{1}\right]$ as $\mathbb{C}_{1}$ and contains only odd-weight codewords. Next, let $\mathbb{C}_{0}^{*}$ be 
an $\left[n, M_{0}^{*}, d_{0}^{*}\right]$ binary code with $d_{0}^{*} \geq d_{0}$, and assume that $\mathbb{C}_{0}^{*}$ is orthogonal to the complement code of $\mathbb{C}_{0}$. That is $\mathbb{C}_{0}^{*} \subseteq\left(\mathbf{1}+\mathbb{C}_{0}\right)^{\perp}$. Now, consider two $n$-dimensional packings $\mathcal{J}_{e}=2 \Lambda$ and $\mathcal{J}_{o}$, given by

$$
\begin{aligned}
\mathcal{J}_{e} & =\mathbf{0}+2 \mathbb{C}_{0}+4 \mathbb{C}_{1}+8 \mathbb{C}_{2}+\cdots+2^{m} \mathbb{C}_{m-1}+2^{m+1} \mathbb{Z}^{n}, \\
\mathcal{J}_{o} & =\mathbf{1}+2 \mathbb{C}_{0}^{*}+4 \mathbb{C}_{1}^{*}+8 \mathbb{C}_{2}+\cdots+2^{m} \mathbb{C}_{m-1}+2^{m+1} \mathbb{Z}^{n},
\end{aligned}
$$

where, with a slight abuse of notation, we have used $\mathbf{0 , 1}$ to denote the codes $\{\mathbf{0}\},\{\mathbf{1}\} \subset \mathbb{F}_{2}^{n}$. Let $\mathcal{J}=\mathcal{J}_{e} \cup \mathcal{J}_{o}$. We have the following theorem, which is similar to Theorem 1 of [24] and includes it as a special case.

Theorem 1. For $n \geq 4 \mu(\Lambda)-8$, the center density of $\mathcal{J}$ is given by

$$
\delta(\mathcal{J})=\frac{M_{0}+M_{0}^{*}}{M_{0}} \delta(\Lambda),
$$

For $n<4 \mu(\Lambda)-8$, we have

$$
\delta(\mathcal{J}) \geq\left(\frac{n+8}{4 \mu(\Lambda)}\right)^{n / 2} \frac{M_{0}+M_{0}^{*}}{M_{0}} \delta(\Lambda),
$$

and if $\mathbb{C}_{0} \cap \mathbb{C}_{0}^{*} \neq \varnothing$, in particular if $\mathbb{C}_{0}$ and $\mathbb{C}_{0}^{*}$ are linear, equality holds in (6).

Proof. It is obvious by construction that

$$
V(\mathcal{J})=2^{n} \frac{M_{0}}{M_{0}+M_{0}^{*}} V(\Lambda) .
$$

It is also obvious that $\mu\left(\mathcal{J}_{e}\right)=4 \mu(\Lambda)$ and $\mu\left(\mathcal{J}_{o}\right) \geq 4 \mu(\Lambda)$, since $d_{0}^{*} \geq d_{0}$. We now show that for any $\alpha \in \mathcal{J}_{e}$ and $\beta \in \mathcal{J}_{o}$, we have

$$
\|\alpha-\beta\|^{2} \geq n+8 .
$$

Let $a, a^{\prime} \in \mathbb{F}_{2}^{n}$ denote the 2's and 4's rows, respectively, in the coordinate array of $\alpha$, and let $b, b^{\prime} \in \mathbb{F}_{2}^{n}$ be the corresponding rows in the coordinate array of $\beta$. We have

$$
a \in \mathbb{C}_{0}, \quad b \in \mathbb{C}_{0}^{*} ; \quad a^{\prime} \in \mathbb{C}_{1}, \quad b^{\prime} \in \mathbb{C}_{1}^{*} ;
$$

and $(\bar{a}, b)=0$, where $\bar{a}=\mathbf{1}+a$ is the complement of $a$, by the definition of $\mathbb{C}_{0}^{*}$. Now let $\xi=\left(\xi_{1}, \ldots, \xi_{n}\right)=\beta-\alpha$. Observe that all the coordinates of $\xi$ are odd integers, and therefore $\|\alpha-\beta\|^{2}=\|\xi\|^{2} \geq n$. Furthermore, if $\xi_{i} \neq \pm 1$ for at least one $i$, then $\|\xi\|^{2} \geq n+8$. Hence assume to the contrary that $\xi=( \pm 1, \ldots, \pm 1)$, and consider the coordinate arrays of $\alpha$ and $\beta=\alpha+\xi$. It can be readily verified (cf. [24, p.122]) that, in this case, the 4's row of $\beta$ is obtained from the 4's row of $\alpha$ by complementing it at those positions where $a_{i}=0$ and $b_{i}=1$. Since $(\bar{a}, b)=0$, the number of such positions must be even. This is a contradiction, as $a^{\prime}$ and $b^{\prime}$ have different parities by construction. This establishes (8) and implies that

$$
\mu(\mathcal{J})=\min _{\substack{\alpha \neq \beta \\ \alpha, \beta \in \mathcal{J}}}\|\alpha-\beta\|^{2} \geq \min \left\{\mu\left(\mathcal{J}_{e}\right), \mu\left(\mathcal{J}_{o}\right), n+8\right\} .
$$

If $n+8 \geq 4 \mu(\Lambda)$, then the minimum in (9) is attained at $\mu\left(\mathcal{J}_{e}\right)=4 \mu(\Lambda)$ and (9) holds with equality. Combining this with (7) establishes (5). If $n+8<4 \mu(\Lambda)$, then the minimum in (9) is attained at $n+8$, which establishes (6). Finally, if $\mathbb{C}_{0} \cap \mathbb{C}_{0}^{*} \neq \varnothing$, we can take $\alpha=2 a+4 a^{\prime}+8 a^{\prime \prime}+\cdots \in \mathcal{J}_{e}$ with $a \in \mathbb{C}_{0} \cap \mathbb{C}_{0}^{*}$ and $a_{1}^{\prime}=1$, where we assume w.l.o.g. that not all the codewords of $\mathbb{C}_{1}$ have a zero in the first position. Then for $\xi=(-3,1,1, \ldots, 1)$, we have $\beta=\alpha+\xi \in \mathcal{J}_{o}$, and both (9) and (6) hold with equality. 
The union $\mathcal{J}$ of $\mathcal{J}_{e}$ and $\mathcal{J}_{o}$ in (4) is, in general, a nonlattice packing. However, if $\Lambda$ is a lattice and the code $\mathbb{C}_{0}$ is self-complementary and self-orthogonal, then the construction of (4), with $\mathbb{C}_{0}^{*}=\mathbb{C}_{0}$, is essentially equivalent to taking the union of $2 \Lambda$ and $(-3,1,1, \ldots, 1)+2 \Lambda$. This, of course, produces a lattice.

Example 1. Let $\mathcal{C}_{24}$ be the $(24,12,8)$ binary Golay code [20, p.65], and let $\mathcal{E}_{24}$ denote the $(24,23,2)$ even-weight binary code consisting of all the even-weight vectors in $\mathbb{F}_{2}^{24}$. Consider the so-called half Leech lattice $H_{24}=\mathcal{C}_{24}+2 \mathcal{E}_{24}+4 \mathbb{Z}^{24}$ with $\mu\left(H_{24}\right)=8$ and $\delta\left(H_{24}\right)=1 / 2$. It is well known that the code $\mathbb{C}_{0}=\mathcal{C}_{24}$ is self-complementary and self-dual, and hence we can take $\mathbb{C}_{0}^{*}=\mathbb{C}_{0}=\mathcal{C}_{24}$. The construction of (4) then produces the lattice

$$
\mathcal{J}_{24}=2 H_{24} \cup(-3,1,1, \ldots, 1)+2 H_{24} .
$$

Since in this case $n=4 \mu\left(H_{24}\right)-8$ and $M_{0}^{*}=M_{0}$, the center density of $\mathcal{J}_{24}$ is $2 \delta\left(H_{24}\right)=1$ by Theorem 1 . This is, of course, the famous Leech lattice, first discovered in [16].

Example 2. Two orthogonal $(20,9,7)$ binary linear codes $\mathcal{C}_{20}$ and $\mathcal{C}_{20}^{*}$ were constructed in [24]. Set $\mathbb{C}_{0}=\mathbf{1}+\mathcal{C}_{20}$ and consider the coordinate-array packing $\Omega_{20}=\mathbb{C}_{0}+2 \mathcal{E}_{20}+4 \mathbb{Z}^{20}$ with $\mu\left(\Omega_{20}\right)=7$ and $\delta\left(\Omega_{20}\right)=7^{10} / 2^{32}$. We again have $n=4 \mu\left(\Omega_{20}\right)-8$. Taking $\mathbb{C}_{0}^{*}=\mathcal{C}_{20}^{*}$ in the construction of (4) produces the nonlattice packing $\mathcal{J}_{20}$ whose center density is twice that of $\Omega_{20}$ by Theorem 1, namely $\delta\left(\mathcal{J}_{20}\right)=2 \delta\left(\Omega_{20}\right)=7^{10} / 2^{31}$. This is the densest known sphere packing in $\mathbb{R}^{20}$ constructed in [24].

Example 2 demonstrates that the construction of [24] is a special case of Theorem 1. In the next section, we show that Theorem 1 also gives rise to several other sphere packings that are denser than any previously known packing.

\section{NEW SPHERE PACKINGS IN DIMENSIONS $n \leq 31$}

In this section, we apply Theorem 1 in dimensions $n=25,26, \ldots, 31$ and compare the densities of the resulting packings to that of the laminated lattices [5, pp.157-180], Bacher lattices [1], and Quebbemann [21] lattices in the corresponding dimensions.

We let $\Omega_{25}, \Omega_{26}, \ldots, \Omega_{31}$ denote the densest known coordinate-array packings in dimensions $n=25,26, \ldots, 31$, respectively. All these packings have the structure

$$
\Omega_{n}=\mathbb{C}_{0}+2 \mathcal{E}_{n}+4 \mathbb{Z}^{n} \quad \text { for } n=25,26, \ldots, 31,
$$

where $\mathcal{E}_{n}$ is the $(n, n-1,2)$ even-weight code, and $\mathbb{C}_{0}$ is (the complement of) a binary code of length $n$ and minimum distance $d_{0}=8$, with the largest known number of codewords. The parameters of these packings are summarized in Table 1. In each case, $\mu\left(\Omega_{n}\right)=8$ and $n \geq 4 \mu\left(\Omega_{n}\right)-8=24$, so that equation (5) of Theorem 1 applies. We shall see that the density of all but one of these packings can be doubled using the construction of (4), and the density of $\Omega_{31}$ can be increased ${ }^{2}$ by a factor of $3 / 2$.

\footnotetext{
${ }^{2}$ In fact, there exists a coordinate-array packing in $\mathbb{R}^{31}$ with center density 1 . However, its density cannot be further increased using the construction of (4).
} 
TABLE 1. Density doubling in dimensions $n=25,26, \ldots, 31$

\begin{tabular}{|c|c|c|c|c|c|c|c|c|}
\hline \multicolumn{4}{|c|}{ Coordinate-array packings } & \multicolumn{3}{|c|}{ Density doubling } & \\
\hline $\mathbb{C}_{0}$ & $(n, k, d)$ & $V\left(\Omega_{n}\right.$ & $\delta\left(\Omega_{n}\right)$ & $\mathbb{C}_{0}^{*}$ & $(n, k, d)$ & $\delta\left(\mathcal{J}_{n}\right)$ & name & $\begin{array}{c}\text { center } \\
\text { density }\end{array}$ \\
\hline $\mathbf{1}+\mathcal{C}_{25}$ & $(25,12,8)$ & $2^{14}$ & $\frac{\sqrt{2}}{4}$ & $\mathcal{C}_{25}$ & $(25,12,8)$ & $\frac{\sqrt{2}}{2}=0.7071 .$. & $\Lambda_{25}$ & $\frac{\sqrt{2}}{2}=0.7071 .$. \\
\hline $\mathbf{1}+\mathcal{C}_{26}$ & $(26,12,8)$ & $2^{15}$ & $\frac{1}{4}$ & $\mathcal{C}_{26}$ & $(26,12,8)$ & $\frac{1}{2}$ & $\Lambda_{26}$ & $\frac{\sqrt{3}}{3}=0.5773 .$. \\
\hline $1+\mathcal{C}_{27}$ & $(27,13,8)$ & $2^{15}$ & $\frac{\sqrt{2}}{4}$ & $\mathcal{C}_{27}^{*}$ & $(27,13,8)$ & $\frac{\sqrt{2}}{2}=0.7071 .$. & $\Lambda_{27}$ & $\frac{1}{2}$ \\
\hline $\mathcal{C}_{28}$ & $(28,14,8)$ & $2^{15}$ & $\frac{1}{2}$ & $\mathcal{C}_{28}^{\perp}$ & $(28,14,8)$ & 1 & $B_{28}$ & $\frac{\sqrt{3}}{3}=0.5773 .$. \\
\hline $\mathbf{1}+\mathcal{C}_{29}$ & $(29,14,8)$ & $2^{16}$ & $\frac{\sqrt{2}}{4}$ & $\mathcal{C}_{29}^{*}$ & $(29,14,8)$ & $\frac{\sqrt{2}}{2}=0.7071 .$. & $B_{29}$ & $\frac{\sqrt{3}}{3}=0.5773 .$. \\
\hline $\mathcal{C}_{30}$ & $(30,15,8)$ & $2^{16}$ & $\frac{1}{2}$ & $\mathcal{C}_{30}^{\perp}$ & $(30,15,8)$ & 1 & $Q_{30}$ & $\frac{3^{13} \sqrt{3}}{2^{22}}=0.6583$. \\
\hline $\mathbf{1}+\mathcal{C}_{31}$ & $(31,16,8)$ & $2^{16}$ & $\frac{\sqrt{2}}{2}$ & $\mathcal{C}_{31}^{\perp}$ & $(31,15,8)$ & $\frac{3 \sqrt{2}}{4}=1.0606 .$. & $Q_{31}$ & $\frac{3^{15}}{2^{23} \sqrt{2}}=1.2095$. \\
\hline
\end{tabular}

We let $\mathcal{C}_{25}, \mathcal{C}_{26}, \ldots, \mathcal{C}_{31}$ denote the best known $[n, M, 8]$ binary codes, for $n=$ $25,26, \ldots, 31$, respectively. We refer to the table of [19] for the parameters of these codes. As it turns out, all of $\mathcal{C}_{25}, \mathcal{C}_{26}, \ldots, \mathcal{C}_{31}$ are linear $(n, k, 8)$ codes. Furthermore, $\mathcal{C}_{27}, \mathcal{C}_{28}, \ldots, \mathcal{C}_{31}$ can be constructed from double-circulant codes [20, pp.505-509]. We shall exploit the properties of double-circulant codes in what follows.

Dimensions 25 and 26. Here, $\mathcal{C}_{25}$ and $\mathcal{C}_{26}$ have parameters $(25,12,8)$ and $(26,12,8)$, respectively. These codes may be obtained straightforwardly from the $(24,12,8)$ Golay code $\mathcal{C}_{24}$ by appending one or two zeros to each codeword. Since $\mathcal{C}_{24}$ is self-dual, it is obvious that $\mathcal{C}_{25}$ and $\mathcal{C}_{26}$ are self-orthogonal. Thus, in the construction of (4), we can take $\mathbb{C}_{0}=\mathbf{1}+\mathcal{C}_{n}$ and $\mathbb{C}_{0}^{*}=\mathcal{C}_{n}$ for $n=25,26$. By Theorem 1 , this doubles the density of $\Omega_{25}$ and $\Omega_{26}$. The resulting packings $\mathcal{J}_{25}$ and $\mathcal{J}_{26}$ have center densities $\sqrt{2} / 2$ and $1 / 2$. Notably, $\mathcal{J}_{25}$ is just a translate of the laminated lattice $\Lambda_{25}$ by the vector $(0,0, \ldots, 2)$.

Dimensions 27, 28, and 29. Here $\mathcal{C}_{28}$ is the $(28,14,8)$ double-circulant code, discovered by Karlin [13]. The generator matrix of $\mathcal{C}_{28}$ has the form

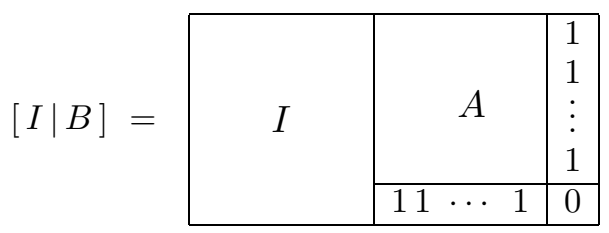

where $A$ denotes a $13 \times 13$ circulant matrix with first row $a=\left(a_{0}, a_{1}, \ldots, a_{12}\right)=$ (010110001101). The nonzeros in $a$ correspond to the nonzero quadratic residues modulo 13; the remaining rows of $A$ are cyclic shifts of $a$. Note that $A$ is a symmetric matrix, and hence so is $B$.

It is easy to see that $\mathcal{C}_{28}$ is self-complementary, but not self-orthogonal. The dual code $\mathcal{C}_{28}^{\perp}$ is generated by $\left[B^{T} \mid I\right]$, and since $B^{T}=B$ it is equivalent to the primal code $\mathcal{C}_{28}$. Indeed, the permutation $i \rightarrow(i+14) \bmod 28 \operatorname{maps} \mathcal{C}_{28}$ and $\mathcal{C}_{28}^{\perp}$ upon each other. Thus, in the construction of (4), we can take $\mathbb{C}_{0}=\mathcal{C}_{28}$ and $\mathbb{C}_{0}^{*}=\mathcal{C}_{28}^{\perp}$. This doubles the density of $\Omega_{28}$ and produces the nonlattice sphere packing $\mathcal{J}_{28}$ with center density 1 . This packing is twice as dense as a laminated lattice $\Lambda_{28}$, 
and is denser than the Bacher lattice $B_{28}$, the best previously known packing in $\mathbb{R}^{28}$.

In dimension 27 , we proceed as follows. The $(27,13,8)$ code $\mathcal{C}_{27}$ can be obtained by shortening $\mathcal{C}_{28}$ in any position. The dual code of $\mathcal{C}_{27}$ is then obtained by puncturing $\mathcal{C}_{28}^{\perp}$ in the corresponding position, since puncturing and shortening are dual operations (cf. [20, pp.27-31]). As $\mathcal{C}_{28}^{\perp}$ has parameters $(28,14,8)$, it follows that $\mathcal{C}_{27}^{\perp}$ has parameters $(27,14,7)$. The even-weight subcode of $\mathcal{C}_{27}^{\perp}$, namely $\mathcal{C}_{27}^{*}=\mathcal{E}_{27} \cap \mathcal{C}_{27}^{\perp}$, is then a $(27,13,8)$ code orthogonal to $\mathcal{C}_{27}$. Substituting $\mathbb{C}_{0}=\mathbf{1}+\mathcal{C}_{27}$ and $\mathbb{C}_{0}^{*}=\mathcal{C}_{27}^{*}$ doubles the density of $\Omega_{27}$ and produces the nonlattice packing $\mathcal{J}_{27}$ with center density $\sqrt{2} / 2$. This is denser than the best previously known packing $\Lambda_{27}$ whose center density is $1 / 2$.

Finally, for $n=29$, the $(29,14,8)$ code $\mathcal{C}_{29}$ can be obtained by appending a zero to each codeword of the $(28,14,8)$ code $\mathcal{C}_{28}$. Appending a zero to each codeword of $\mathcal{C}_{28}^{\perp}$ we obtain a $(29,14,8)$ code $\mathcal{C}_{29}^{*}$ orthogonal to $\mathcal{C}_{29}$. Setting $\mathbb{C}_{0}=\mathbf{1}+\mathcal{C}_{29}$ and $\mathbb{C}_{0}^{*}=\mathcal{C}_{29}^{*}$ in (4) doubles the density of $\Omega_{29}$ and produces the nonlattice packing $\mathcal{J}_{29}$ with center density $\sqrt{2} / 2$. This packing is again denser than any previously known packing in $\mathbb{R}^{29}$.

Dimensions 30 and 31. Here $\mathcal{C}_{30}$ is the $(30,15,8)$ double-circulant code discovered by Karlin [13], and generated by $[I \mid A]$, where $A$ is a $15 \times 15$ circulant matrix with first row $\left(a_{0}, a_{1}, \ldots, a_{14}\right)=(01001111010010)$. Since $w t(a)=7$, this code is self-complementary, although it is not self-orthogonal. However, it is known [20, p.501] that the dual of a double-circulant code of this type is equivalent to the primal code. (Indeed, we have $A=\left[a_{i, j}\right]=\left[a_{(j-i) \bmod 14}\right]$ so that $A^{T}=\left[a_{j, i}\right]=\left[a_{(i-j) \bmod 14}\right]$. Hence the reverse order permutation $i \rightarrow 31-i$ maps $\mathcal{C}_{30}$ and $\mathcal{C}_{30}^{\perp}$ upon each other.) It follows that in the construction of (4) we can set $\mathbb{C}_{0}=\mathcal{C}_{30}$ and $\mathbb{C}_{0}^{*}=\mathcal{C}_{30}$. This doubles the density of $\Omega_{30}$ and produces the nonlattice packing $\mathcal{J}_{30}$ with center density 1 . This is again denser than the best previously known packing in $\mathbb{R}^{30}$ - the Quebbemann lattice $Q_{30}$, whose center density is $3^{13} \sqrt{3} / 2^{22}=0.6583 \ldots$

For $n=31$, the best known code $\mathcal{C}_{31}$ has parameters $(31,16,8)$ and is generated by $[I \mid \widetilde{A}]$, where $\widetilde{A}$ is the $16 \times 15$ matrix obtained by appending the all-one row to the circulant matrix $A$. Hence, the dual code of $\mathcal{C}_{31}$ is generated by $\left[\widetilde{A}^{T} \mid I\right]=$ $\left[A^{T}\left|\mathbf{1}^{T}\right| I\right]$, where $\mathbf{1}^{T}$ denotes the all-one column of length 15 . Since the matrix $\left[A^{T} \mid I\right]$ generates a $(30,15,8)$ code, as we have seen in the foregoing paragraph, it is clear that $\mathcal{C}_{31}^{\perp}$ is a $(31,15,8)$ code. Thus, we can take $\mathbb{C}_{0}=\mathbf{1}+\mathcal{C}_{31}$ and $\mathbb{C}_{0}^{*}=\mathcal{C}_{31}^{\perp}$ in the construction of (4). By equation (5) of Theorem 1, this increases the density of $\Omega_{31}$ by a factor of $3 / 2$ (it is clear that the density of $\Omega_{31}$ cannot be doubled, since $\operatorname{dim} \mathcal{C}_{31}>31 / 2$ ) and produces the nonlattice packing $\mathcal{J}_{31}$ with center density $3 \sqrt{2} / 4=1.0606 \ldots$ This packing is 1.5 times denser than a laminated lattice $\Lambda_{31}$, but not as dense as the Quebbemann lattice $Q_{31}$ whose center density is $3^{15} / 2^{23} \sqrt{2}=1.2095 \ldots$

The center densities of the packings $\mathcal{J}_{25}, \mathcal{J}_{26}, \ldots, \mathcal{J}_{31}$ constructed herein are listed in Table 1 . We note that there exist several inequivalent codes with the same parameters as $\mathcal{C}_{25}, \mathcal{C}_{26}, \ldots, \mathcal{C}_{31}$. For instance, codes with the same parameters as $\mathcal{C}_{29}, \mathcal{C}_{30}, \mathcal{C}_{31}$ can be obtained by shortening the $(32,17,8)$ code found by Cheng and Sloane [5, p.235]. It is likely, therefore, that there exist other inequivalent sphere packings with the same density as $\mathcal{J}_{25}, \mathcal{J}_{26}, \ldots, \mathcal{J}_{31}$. However, the double-circulant constructions, employed in this section, seem to be the most convenient for our purposes. 


\section{NEW SPHERE PACKINGS IN DIMENSIONS $n \leq 63$}

We now examine density doubling in higher dimensions. As we shall see, the construction of (4) produces dense sphere packings when the dimension $n$ is just below $2^{6}=64$, in particular for $n=55,56, \ldots, 63$. As before, we let $\Omega_{n}$ denote the densest known coordinate array packing in dimension $n$. For $n=49,50, \ldots, 63$, all these packings have the structure

$$
\Omega_{n}=\mathbb{C}_{0}+2 \mathcal{H}_{n}+4 \mathbb{Z}^{n}
$$

where $\mathcal{H}_{n}$ is the $(n, n-7,4)$ code obtained by shortening the $(64,57,4)$ extended Hamming code $\left[20\right.$, p.23], and $\mathbb{C}_{0}$ is (the complement of) the best known $[n, M, 16]$ binary code, denoted $\mathcal{C}_{n}$. Referring to the table of [19], we see that all of $\mathcal{C}_{49}, \ldots$, $\mathcal{C}_{63}$ are again linear $(n, k, 16)$ codes.

Dimensions $\mathbf{5 7}, \mathbf{5 8}, \ldots, \mathbf{6 3}$. Here $\mathcal{C}_{63}$ is the $(63,27,16)$ primitive cyclic code, discovered by Kasami and Tokura [14], with zeros at $\alpha^{0}, \alpha^{1}, \alpha^{3}, \alpha^{5}, \alpha^{7}, \alpha^{9}, \alpha^{11}, \alpha^{21}$, where $\alpha$ is a primitive element of $\mathbb{F}_{64}$. For the theory of cyclic codes, see $[20$, pp.188255]. The dual code $\mathcal{C}_{63}^{\perp}$ has zeros at $\alpha^{1}, \alpha^{3}, \alpha^{5}, \alpha^{9}, \alpha^{11}$ and therefore contains $\mathcal{C}_{63}$ as a subcode. We conclude that $\mathcal{C}_{63}$ is self-orthogonal, although it is obviously not self-complementary. The codes $\mathcal{C}_{57}, \mathcal{C}_{58}, \ldots, \mathcal{C}_{62}$ with parameters $(n, n-36,16)$ can be obtained by shortening $\mathcal{C}_{63}$. Clearly, all these codes are self-orthogonal, and none of them is self-complementary.

It is now easy to see that for $n=57,58, \ldots, 63$, we have $\mu\left(\Omega_{n}\right)=16$ and $V\left(\Omega_{n}\right)=2^{36} \cdot 2^{7}$, so that $\delta\left(\Omega_{n}\right)=\mu\left(\Omega_{n}\right)^{n / 2} / 2^{n} V\left(\Omega_{n}\right)=2^{n-43}$. As $n \geq 4 \mu\left(\Omega_{n}\right)-$ $8=56$, equation (5) of Theorem 1 applies. Hence the construction of (4) with $\mathbb{C}_{0}=\mathbf{1}+\mathcal{C}_{n}$ and $\mathbb{C}_{0}^{*}=\mathcal{C}_{n}$ doubles the density of $\Omega_{n}$ and produces the sphere packing $\mathcal{J}_{n}$ with center density $2^{n-42}$.

In particular, for $n=60$ we have $\delta\left(\mathcal{J}_{60}\right)=2^{18}$. This is denser than the previous record in $\mathbb{R}^{60}$ reported in [5, p.xvi], namely the Kschischang and Pasupathy [15] lattice, constructed using partitions of the Eisenstein integers, with center density $3^{11}=2^{17.43 . .}$. However, we have been recently informed by N.D. Elkies [10] that a lattice denser than $\mathcal{J}_{60}$ can be obtained as a cross-section of his 64-dimensional lattice $E_{64}$. In fact, the cross-sections $E_{n}$ of the Elkies [8,9] lattice $E_{64}$ have center densities of at least

$$
\begin{array}{rccccccc}
n: & 63 & 62 & 61 & 60 & 59 & 58 & 57 \\
\log _{2} \delta\left(E_{n}\right): & 23.92 . . & 22.13 . . & 20.54 . . & 19.04 . . & 17.75 . . & 16.46 . . & 15.37 . .
\end{array}
$$

Thus, the Elkies lattices [10] are invariably denser than $\mathcal{J}_{57}, \mathcal{J}_{58}, \ldots, \mathcal{J}_{63}$, but only slightly so for $n=57,58,59$.

Dimensions 55 and 56. Here $\mathcal{C}_{55}$ is the $(55,20,16)$ cyclic code found by Berlekamp [3]. It has zeros at $\alpha^{0}, \alpha^{1}, \alpha^{5}, \alpha^{11}$, where $\alpha$ is an element of order 55 in $\mathbb{F}_{2^{20}}$. The zeros of the dual code are at $\alpha^{0}$ and $\alpha^{1}$, which implies that $\mathcal{C}_{55}$ is selforthogonal but not self-complementary. The $(56,21,16)$ code $\mathcal{C}_{56}$ can be obtained by appending an overall parity-check position to the $(55,21,15)$ cyclic code with zeros at $\alpha^{1}, \alpha^{5}, \alpha^{11}$. Thus $\mathcal{C}_{56}$ is generated by

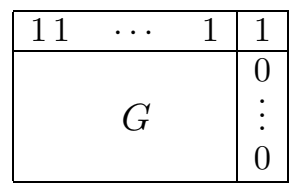


where $G$ is a generator matrix for $\mathcal{C}_{55}$. Since $\mathcal{C}_{55}$ is self-orthogonal and contains only even-weight codewords, it follows that $\mathcal{C}_{56}$ is self-orthogonal and selfcomplementary. For $n=56$ we have $\mu\left(\Omega_{56}\right)-8=n$, so that equation (5) still applies. Thus, setting $\mathbb{C}_{0}=\mathbb{C}_{0}^{*}=\mathcal{C}_{56}$ in the construction of (4) produces a packing with center density $\delta\left(\mathcal{J}_{56}\right)=2 \delta\left(\Omega_{56}\right)=2^{15}$. For $n=55$, we have $\delta\left(\Omega_{55}\right)=2^{13}$. However, the density of $\Omega_{55}$ can no longer be doubled, although it can be increased. Setting $\mathbb{C}_{0}=\mathbf{1}+\mathcal{C}_{55}$ and $\mathbb{C}_{0}^{*}=\mathcal{C}_{55}$ in the construction of (4), and using equation (6) of Theorem 1, we obtain the sphere packing $\mathcal{J}_{55}$ with center density $(63 / 64)^{27.5} 2^{14}>2^{13}$. (It is easy to see that $(6)$ holds with equality in this case. For instance, consider $(2,2, \ldots, 2) \in \mathcal{J}_{e}$ and $(5,1, \ldots, 1) \in \mathcal{J}_{o}$.) Neither $\mathcal{J}_{56}$ nor $\mathcal{J}_{55}$ is as dense as as the Elkies-Shioda $[8,23]$ lattice in 56 dimensions, or as the packings obtained by laminating the Elkies lattice $E_{54}$ [10].

The construction of (4) does not appear to be useful for dimensions $n<55$. For instance, for $n=54$, we have

$$
2\left(\frac{n+8}{4 \mu\left(\Omega_{n}\right)}\right)^{n / 2}=2\left(\frac{62}{64}\right)^{27}<1,
$$

so that $\Omega_{54}$ is denser than $\mathcal{J}_{54}$. Of course, it is still possible to double the density of a coordinate-array packing $\Lambda$ with $\mu(\Lambda) \leq 15$. However, in dimensions $n=$ $32,33, \ldots, 63$ such packings are distinctly inferior to coordinate-array packings with $\mu(\Lambda)=16$. For example, for $n=52$, taking a $(52,18,15)$ code as $\mathbb{C}_{0}$ in $(10)$ produces a packing with center density $15^{26} / 2^{93}=382.45 \ldots$ Even if we were to double the density of this packing, the result would still be inferior to $\delta\left(\Omega_{52}\right)=2^{10}$. This phenomenon may be attributed to the fact that the natural choice of parameters in the coordinate-array construction of (1) is such that $\mu(\Lambda)$ is a power of 2 (see the remark following (3) and [5, p.150]).

Thus, we see that in general the construction of (4) would be most successful when $n+8$ is close to a power of 2 , with the cases $n=18,20$ being notable exceptions $[4,24]$. On the other hand, as the dimension $n$ grows the coordinatearray construction (1) itself becomes increasingly less attractive. For example, the best known coordinate-array packing in 128 dimensions has center density $2^{84}$, whereas the Elkies [8] lattice $E_{128}$ has center density $2^{97.40 . .}$. Thus, it is not clear whether it would be worthwhile to straightforwardly pursue the ideas of (4) for $n=120,121, \ldots, 127$, for instance.

One promising way to extend our approach to higher dimensions would be to generalize the construction of (4) to (non-binary) partitions over the Eisenstein, Hurwitz, and Cayley integer rings. It is clear that coordinate-arrays can be defined modulo any prime, and the notation of (1) extends in the obvious way. For instance, $\phi=1+i$ is a prime in the ring $\mathbb{H}$ of Hurwitz quaternionic integers, and the quaternionic version of the so-called quarter-Leech lattice may be constructed as

$$
Q_{24}=H_{6}+\phi S_{6}+\phi^{2} \mathbb{H}^{6},
$$

where $H_{6}$ is the $(6,3,4)$ quaternary hexacode $[5, \mathrm{p} .301]$ and $S_{6}$ is the $(6,5,2)$ zerosum code over $\mathbb{F}_{4}$. It can be easily shown that the density of $Q_{24}$ can be quadrupled, in a manner similar to (4), to produce the Leech lattice $\Lambda_{24}$. In general, it is possible to show that the density of a quaternionic packing of the type $\Lambda=\mathbb{C}_{0}+\phi S_{n}+\phi^{2} \mathbb{H}^{n}$ can be quadrupled provided $\mathbb{C}_{0}$ is self-orthogonal under the Hermitian inner product in $\mathbb{F}_{4}$ and contains only even-weight codewords. A similar construction produces 
the quaternionic version of the packing $\mathcal{J}_{20}$ (cf. [24]). These ideas may lead to further improvements on the best known density of sphere packings in $\mathbb{R}^{n}$.

\section{TABLE OF DENSEST KNOWN SPHERE PACKINGS}

A table of the densest known sphere packings in $\mathbb{R}^{n}$ for the first 24 dimensions may be found in Conway and Sloane [5, p.15]; it is extended in a graphical form up to $n \leq 48$ in Figure 1.5 of [5, p.14].

However, with the recent results of [24], [6], [10], [4] and those obtained herein, the table of [5] is now out of date for 13 out of the 48 dimensions, namely for

$$
n=18,20,22,27,28,29,30,33,34,44,45,46,47 .
$$

We therefore believe that it would be useful to have an updated table of the densest known sphere packings in dimensions $n \leq 48$ available in the literature. Such a table is presented below. The center density of the laminated lattices is also shown in our table. 
DENSITY DOUBLING, DOUBLE-CIRCULANTS, AND NEW SPHERE PACKINGS 281

TABLE 2. Densest known sphere packings in dimensions $n=1,2, \ldots, 48$

\begin{tabular}{|c|c|c|c|c|}
\hline \multicolumn{4}{|c|}{ Best known } & \multirow{2}{*}{$\begin{array}{c}\text { Laminated } \\
\text { center } \\
\text { density }\end{array}$} \\
\hline$n$ & $\begin{array}{l}\text { center } \\
\text { density }\end{array}$ & Name & Reference & \\
\hline 1 & $\frac{1}{2}=0.50000$ & $\mathbb{Z}=\Lambda_{1}$ & {$[5$, p. 106$]$} & $\frac{1}{2}=0.50000$ \\
\hline 2 & $\frac{1}{2 \sqrt{3}}=0.28868$. & $A_{2}=\Lambda_{2}$ & {$[5, \mathrm{p} .110]$} & $\frac{1}{2 \sqrt{3}}=0.28868 .$. \\
\hline 3 & $\frac{1}{4 \sqrt{2}}=0.17678$. & $A_{3}=\Lambda_{3}$ & {$[5, \mathrm{p} .112]$} & $\frac{1}{4 \sqrt{2}}=0.17678 .$. \\
\hline 4 & $\frac{1}{8}=0.12500$ & $D_{4}=\Lambda_{4}$ & {$[5, \mathrm{p} .118]$} & $\frac{1}{8}=0.12500$ \\
\hline 5 & $\frac{1}{8 \sqrt{2}}=0.08839$. & $D_{5}=\Lambda_{5}$ & {$[5$, p.117] } & $\frac{1}{8 \sqrt{2}}=0.08839 .$. \\
\hline 6 & $\frac{1}{8 \sqrt{3}}=0.07217 .$. & $E_{6}=\Lambda_{6}$ & {$[5, \mathrm{p} .125]$} & $\frac{1}{8 \sqrt{3}}=0.07217 .$. \\
\hline 7 & $\frac{1}{16}=0.06250$ & $E_{7}=\Lambda_{7}$ & {$[5, \mathrm{p} .124]$} & $\frac{1}{16}=0.06250$ \\
\hline 8 & $\frac{1}{16}=0.06250$ & $E_{8}=\Lambda_{8}$ & {$[5$, p. 120$]$} & $\frac{1}{16}=0.06250$ \\
\hline 9 & $\frac{1}{16 \sqrt{2}}=0.04419 .$. & $\Lambda_{9}$ & {$[5, \mathrm{p} .170]$} & $\frac{1}{16 \sqrt{2}}=0.04419 .$. \\
\hline 10 & $\frac{5}{128}=0.03906$ & $P_{10 c}$ & {$[5, \mathrm{p} .140]$} & $\frac{1}{16 \sqrt{3}}=0.03608$. \\
\hline 11 & $\frac{9}{256}=0.03516 .$. & $P_{11 a}$ & {$[5$, p.140] } & $\frac{1}{32}=0.03125$ \\
\hline 12 & $\frac{1}{27}=0.03704$. & $K_{12}$ & {$[5, \mathrm{p} .127]$} & $\frac{1}{32}=0.03125$ \\
\hline 13 & $\frac{9}{256}=0.03516$. & $P_{13 a}$ & {$[5, \mathrm{p} .144]$} & $\frac{1}{32}=0.03125$ \\
\hline 14 & $\frac{1}{16 \sqrt{3}}=0.03608$. & $\Lambda_{14}$ & {$[5, \mathrm{p} .170]$} & $\frac{1}{16 \sqrt{3}}=0.03608 .$. \\
\hline 15 & $\frac{1}{16 \sqrt{2}}=0.04419 .$. & $\Lambda_{15}$ & {$[5, \mathrm{p} .170]$} & $\frac{1}{16 \sqrt{2}}=0.04419 .$. \\
\hline 16 & $\frac{1}{16}=0.06250$ & $\Lambda_{16}$ & {$[5$, p. 129$]$} & $\frac{1}{16}=0.06250$ \\
\hline 17 & $\frac{1}{16}=0.06250$ & $\Lambda_{17}$ & {$[5, \mathrm{p} .176]$} & $\frac{1}{16}=0.06250$ \\
\hline 18 & $\frac{3^{9}}{2^{18}}=0.07508$ & $\mathcal{J}_{18}$ & {$[4]$} & $\frac{1}{8 \sqrt{3}}=0.07217 .$. \\
\hline 19 & $\frac{1}{8 \sqrt{2}}=0.08839 .$. & $\Lambda_{19}$ & {$[5, \mathrm{p} .176]$} & $\frac{1}{8 \sqrt{2}}=0.08839 .$. \\
\hline 20 & $\frac{7^{10}}{2^{31}}=0.13154$ & $\mathcal{J}_{20}$ & {$[24]$} & $\frac{1}{8}=0.12500$ \\
\hline 21 & $\frac{1}{4 \sqrt{2}}=0.17678$. & $\Lambda_{21}$ & {$[5, \mathrm{p} .176]$} & $\frac{1}{4 \sqrt{2}}=0.17678 .$. \\
\hline 22 & $\frac{11^{11}}{2^{23} 3^{10} \sqrt{3}}=0.33254$. & $\mathcal{J}_{22}$ & {$[6]$} & $\frac{1}{2 \sqrt{3}}=0.28868 .$. \\
\hline 23 & $\frac{1}{2}=0.50000$ & $\Lambda_{23}$ & {$[5, \mathrm{p} .176]$} & $\frac{1}{2}=0.50000$ \\
\hline 24 & $1=1.00000$ & $\Lambda_{24}$ & {$[16]$} & $1=1.00000$ \\
\hline
\end{tabular}


Table 2 (continued)

\begin{tabular}{|c|c|c|c|c|}
\hline \multirow[b]{2}{*}{$n$} & \multicolumn{3}{|c|}{ Best known } & \multirow{2}{*}{$\begin{array}{c}\text { Laminated } \\
\text { center } \\
\text { density }\end{array}$} \\
\hline & $\begin{array}{l}\text { center } \\
\text { density }\end{array}$ & Name & Reference & \\
\hline 25 & $\frac{1}{\sqrt{2}}=0.70711$. & $\Lambda_{25}$ & {$[5$, p. 177$]$} & $\frac{1}{\sqrt{2}}=0.70711 .$. \\
\hline 26 & $\frac{1}{\sqrt{3}}=0.57735$. & $\Lambda_{26}$ & {$[5, \mathrm{p} .177]$} & $\frac{1}{\sqrt{3}}=0.57735 .$. \\
\hline 27 & $\frac{1}{\sqrt{2}}=0.70711 .$. & $\mathcal{J}_{27}$ & & $\frac{1}{2}=0.50000$ \\
\hline 28 & $1=1.00000$ & $\mathcal{J}_{28}$ & & $\frac{1}{2}=0.50000$ \\
\hline 29 & $\frac{1}{\sqrt{2}}=0.70711 .$. & $\mathcal{J}_{29}$ & & $\frac{1}{2}=0.50000$ \\
\hline 30 & $1=1.00000$ & $\mathcal{J}_{30}$ & & $\frac{1}{\sqrt{3}}=0.57735 .$. \\
\hline 31 & $\frac{3^{15}}{2^{23} \sqrt{2}}=1.20952$. & $Q_{31}, E_{31}$ & {$[21],[10]$} & $\frac{1}{\sqrt{2}}=0.70711 .$. \\
\hline 32 & $\frac{3^{16}}{2^{24}}=2.56578$. & $Q_{32}, Q_{32}^{\prime}, E_{32}, B_{32}$ & {$[21],[22],[8],[2]$} & $1=1.00000$ \\
\hline 33 & $\frac{3^{16} \sqrt{3}}{2^{25}}=2.22203$. & $E_{33}$ & {$[10]$} & $1=1.00000$ \\
\hline 34 & $\frac{3^{16} \sqrt{3}}{2^{25}}=2.22203$. & $E_{34}$ & {$[10]$} & $\frac{2}{\sqrt{3}}=1.15470 .$. \\
\hline 35 & $2 \sqrt{2}=2.82843 .$. & $B_{35}$ & {$[5$, p. 234$]$} & $\sqrt{2}=1.41421 .$. \\
\hline 36 & $\frac{2^{18}}{3^{10}}=4.43943$. & $K s_{36}$ & {$[15]$} & $2=2.0000$ \\
\hline 37 & $2^{2} \sqrt{2}=5.65685$. & $\Omega_{37}$ & {$[5$, p. 150$]$} & $2 \sqrt{2}=2.82842$. \\
\hline 38 & $2^{3}=8.00000$ & $\Omega_{38}$ & {$[5, \mathrm{p} .150]$} & $\frac{8}{\sqrt{3}}=4.61880 .$. \\
\hline 39 & $\frac{3^{16}}{2^{20} \sqrt{14}}=10.9718$. & from $P_{48 p / q}$ & {$[5, \mathrm{p} .167]$} & $8=8.00000$ \\
\hline 40 & $\frac{3^{17}}{2^{22} \sqrt{2}}=21.7714$. & from $P_{48 p / q}$ & {$[5, \mathrm{p} .167]$} & $16=16.0000$ \\
\hline 41 & $\frac{3^{17}}{2^{21} \sqrt{2}}=43.5428$. & from $P_{48 p / q}$ & {$[5, \mathrm{p} .167]$} & $16 \sqrt{2}=22.6274 .$. \\
\hline 42 & $\frac{3^{18}}{2^{22}}=92.3682 .$. & from $P_{48 p / q}$ & {$[5$, p.167] } & $\frac{64}{\sqrt{3}}=36.9504 .$. \\
\hline 43 & $\frac{3^{19}}{2^{22} \sqrt{2}}=195.943 .$. & from $P_{48 p / q}$ & {$[5, \mathrm{p} .167]$} & $64=64.0000$ \\
\hline 44 & $\frac{17^{22}}{3^{24} 2^{43}}=472.799$. & $\mathcal{J}_{44}$ & {$[6]$} & $128=128.000$ \\
\hline 45 & $\frac{17^{22} \sqrt{17}}{3^{24} 2^{44}}=974.700$. & $\mathcal{J}_{45}$ & {$[6]$} & $256=256.000$ \\
\hline 46 & $\frac{13^{23}}{3^{46} \sqrt{3}}=2719.94 .$. & $\mathcal{J}_{46}$ & {$[6]$} & $\frac{1024}{\sqrt{3}}=591.206$. \\
\hline 47 & $\frac{35^{23} \sqrt{35}}{3^{24} 2^{70}}=5788.81$. & $\mathcal{J}_{47}$ & {$[6]$} & $1024 \sqrt{2}=1448.15$ \\
\hline 48 & $\frac{3^{24}}{2^{24}}=16834.1 .$. & $P_{48 p / q}$ & {$[5, \mathrm{p} .149]$} & $4096=4096.00$ \\
\hline
\end{tabular}




\section{ACKNOWLEDGEMENT}

I am indebted to J.H. Conway and N.J.A. Sloane for the preprint of $[6]$ and for their comments on this manuscript. I am grateful to N.D. Elkies for communicating to me his results in $[8,9,10]$, and for valuable discussions. I would like to

thank Jürgen Bierbrauer for the preprint of [4]. Finally, I wish to acknowledge the invaluable help of Hagit Itzkowitz.

\section{REFERENCES}

1. R. Bacher, Dense lattices in dimensions 28 and 29, Invent. Math., 130 (1997), 153-158. MR 98e: 11080

2. C. Bachoc, Voisinage au sens de Kneser pour les réseaux quaternioniens, Comment. Math. Helvetici, 70 (1995), 350-374. MR 96d:11077

3. E.R. Berlekamp, Algebraic Coding Theory, McGraw-Hill, New York, 1968. MR 38:6873

4. J. Bierbrauer and Y. Edel, Dense sphere packings from new codes, J. Algebraic Combinatorics submitted for publication, 1998.

5. J.H. Conway and N.J.A.Sloane, Sphere Packings, Lattices and Groups, second edition, Springer-Verlag, New York, 1993. MR 93h:11069

6. J.H. Conway and N.J.A. Sloane, The antipode construction for sphere packings, Inventiones Math., 123 (1996), 309-313. MR 97a:11109

7. H.S.M. Coxeter and J.A. Todd, An extreme duodenary form, Canad. J. Math., 5 (1953), 384392. MR 14:1066a

8. N.D. Elkies, Mordell-Weil lattices in characteristic 2: Construction and first properties, International Math. Research Notices, 8 (1994), 343-361. MR 95f:11046

9. N.D.Elkies, Mordell-Weil lattices in characteristic 2: the Leech lattice as a Mordell-Weil lattice, Inventiones Math. 128 (1997), 1-8. MR 98c:11063

10. N.D. Elkies, personal communication, February 1996.

11. C.F. Gauss, Besprechung des Buchs von L.A. Seeber: Untersuchungen über die Eigenschaften der positiven ternären quadratischen Formen usw., Göttingsche Gelehrte Anzeigen, July 9, 1831.

12. D. Hilbert, Mathematische Probleme, Archiv. Math. Phys., 1 (1901), 44-63 and 213-237.

13. M. Karlin, New binary coding results by circulants, IEEE Trans. Inform. Theory, 15 (1969), 81-92. MR 40:2425

14. T. Kasami and N. Tokura, Some remarks on BCH bounds and minimum weights of binary primitive BCH codes, IEEE Trans. Inform. Theory, 15 (1969), 408-413.

15. F.R. Kschischang and S. Pasupathy, Some ternary and quaternary codes and associated sphere packings, IEEE Trans. Inform. Theory, 38 (1992), 227-246. MR 93a:94029

16. J. Leech, Notes on sphere packings, Canad. J. Math., 19 (1967), 251-267. MR 35:878

17. J. Leech and N.J.A. Sloane, New sphere packings in dimensions 9-15, Bull. Amer. Math. Soc., 76 (1970), 1006-1010. MR 42:965

18. J. Leech and N.J.A. Sloane, Sphere packings and error-correcting codes, Canad. J. Math., 23 (1971), 718-745. MR 44:3211

19. S.N. Litsyn, Table of best known binary codes, in Handbook of Coding Theory, V. S. Pless and W. C. Huffman (Editors) Amsterdam: Elsevier, 1998.

20. F.J. MacWilliams and N.J.A. Sloane, The Theory of Error-Correcting Codes, North-Holland, New York, 1977. MR 57:5408a

21. H.-G. Quebbemann, A construction of integral lattices, Mathematika, 31 (1984), 137-140. MR 86c: 11044

22. H.-G. Quebbemann, Lattices with theta functions for $G(\sqrt{2})$ and linear codes, J. Algebra, 105 (1987), 443-450. MR 88f: 11063

23. T. Shioda, A Collection: Mordell-Weil Lattices, Max-Planck Institute Math., Bonn, 1991.

24. A. Vardy, A new sphere packing in 20 dimensions, Inventiones Math., 121 (1995), 119-133. MR 96c:52034

Coordinated Science Laboratory, University of Illinois, Urbana, Illinois 61801 Current address: Ecole Supérieure de Science Informatiques, Route des Colles, BP145, 06903 Sophia-Antipolis, France 